\title{
C-Quad: A Miniature, Foldable Quadruped with C-Shaped Compliant Legs
}

\author{
Ahmet Furkan Güç, Mert Ali İhsan Kalın, Cem Karakadıoğlu, and Onur Özcan \\ Bilkent University, Mechanical Engineering Department, Ankara, Turkey
}

\begin{abstract}
C-Quad is an origami-inspired, foldable, miniature robot whose legs and body are all machined from one PET sheet each. The already famous compliant legs are modified such that they can be manufactured from a flat PET sheet and folded into the $\mathrm{C}$-shape wanted. The compliant legs enable the miniature robot to run fast and scale obstacles with ease due to the geometry of the legs. C-Quad has four legs that are manufactured separately from the main body frame, which is also manufactured from a single PET sheet. All of its legs are actuated individually with a total of four DC motors. Despite the thin PET film, the structural rigidity and robustness of the body frame is increased by using specialized folds and locks. The manufacturing and assembly of the robot takes approximately 2.5 hours. C-Quad carries a battery, an Arduino Pro Micro control board, a bluetooth communication module, custom made encoders and commercially available IR sensors for motor speed control and motor drivers, all of which weighs 38 grams. By using very simple control strategies, it can achieve the speed of 2.7 Bodylengths/sec, can perform in-place turns and can climb over obstacles more than half of its height.

Index Terms-Origami-Inspired Robots, Print-and-Fold Robots, Foldable Legged Robots, Unconventional Manufacturing, Miniature Robots.
\end{abstract}

\section{INTRODUCTION}

Miniaturization of robots offers interesting opportunities to the robotics community by decreasing the costs of robots and providing them the ability to explore and inspect confined spaces such as ruins, crash sites and pipelines [1]. On the other hand miniature robotics is still facing big challenges especially due to manufacturing, assembly, actuation and power issues of small robots. Miniature robotics have a variety of manufacturing techniques that suggest solutions to the manufacturing and assembly problems such as laser cutting materials and creating multilayer composite structures in Smart Composite Microstructure (SCM) [2], [3], MEMS fabrication [4] and 3D printing for lightweight robots [5]. All these techniques have advantages and disadvantages such as SCM is very powerful at creating cheap and miniature robots but requires multilayer processing and good alignment between layers, MEMS fabrication can make very small robots however the equipment required are significantly more expensive than the other techniques, and $3 \mathrm{D}$ printing can make very small and lightweight robots but structures are often very rigid and brittle. Origami-inspired (more correctly Kirigami-inspired, since cutting is involved) "foldable robots" technique is another miniature robot manufacturing method that is closely related to the SCM method. This method offers laser-machining single sheets in 2D and folding them into more complex 3D miniature shapes or mechanisms. Using foldable robots technique, we can make

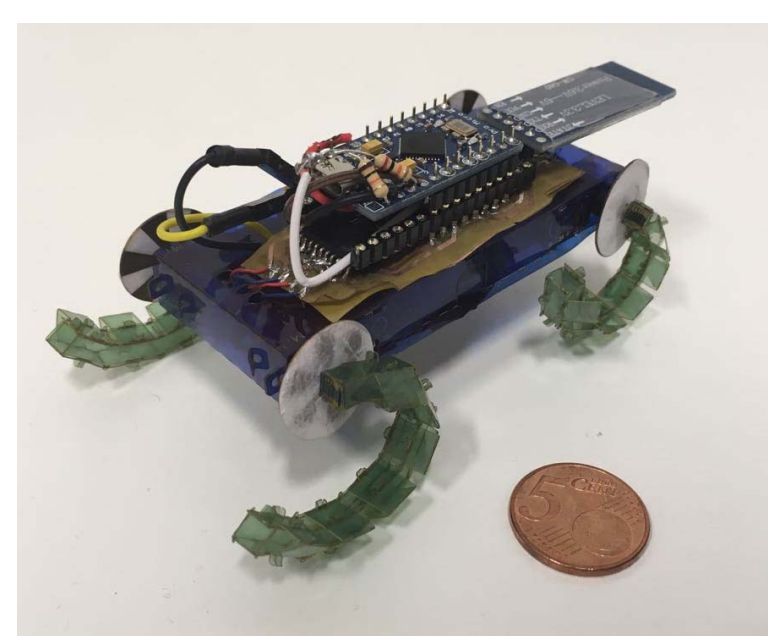

Fig. 1. The foldable, miniature, C-shaped-legged quadruped, C-Quad.

cheaper compliant robots that do not require alignment of multiple layers, however designing the crease patterns can often get very complex. In the design and manufacturing of the C-Quad (Fig. 1), we leveraged this foldable robots technique.

Foldable robots and other SCM and its relative fabrication methods offer compact designs with not only increasing the compliance and robustness or the structures but also enabling cheap and time saving manufacturing and assembly processes. Multilayer fabrication-based SCM and relative processes so far enabled fabrication of many famous miniature robots such as RoACH family of robots [6], [7], DASH [8], and HAMR [9]. The single layer derivative of SCM, the foldable robots technique, was initially proposed as the idea of origami inspired printable robotics [10], and exploited in a variety of works in miniature robotics to grasp [11], [12], fly [13], crawl [14], [15] and walk [16], [17], [18]. Moreover, mechanisms that can fold itself has been developed with the aid of shape-memory sheets [19].

In the design of the C-Quad, we wanted to use a leg design that would provide the robot a high mobility. Originally, RHex showed impressive rough terrain locomotion capabilities using C-shaped compliant legs [20]. More recently a similar leg design is implemented by Maryland Microrobotics Laboratory on a robot much smaller (sub-2 grams) using 3D printed rigid materials, which proved that even without the compliance, $\mathrm{C}$-shaped leg structure can help a miniature robot walk on rough terrain [5]. C-Quad uses a similar Cshaped leg structure that is compliant.

Most foldable robots utilize simple fourbar transmissions that can be manufactured on a single sheet of material in 
order to exploit the ease of manufacturing in this technique. C-Quad is proposed as a design that offers robust structure, fast running, in-place turning, steering and obstacle scaling, with the downside of manufacturing the complete robot in parts on different sheets and a more complicated crease pattern for the leg design. By using a base material of thin PET sheet and optimized folds and locks, the main body frame and each leg structure form part of the highly resilient assembly. The major contribution of this paper is the foldable design of the $\mathrm{C}$-shaped legs and their integration to a miniature robot. The modified C-shape enables fast locomotion by increasing the contact time between the leg and the ground. Main control strategy is based on controlling individual speed of the legs with the aid of IR sensors and custom encoder disks. Moreover, C-Quad has remote control with the aid of a bluetooth serial module along with the micro-controller devices located on a custom flexible printed circuit board (PCB). The resulting untethered robot weighs 38 grams and can run with a speed of 2.7 Bodylengths/sec which correspond to $27.7 \mathrm{~cm} /$ second.

\section{DESIGN OF THE C-QUAD}

\section{A. Body Design, Locks and Folds}

In order to come up with a structure that can sustain required rigidity and robustness, specialized folds become essential. Initially, T-shaped folds, first introduced in [21], are exploited to increase the stiffness of the body structure of the robot. T-folds are out-of-plane extensions that can be added to arbitrary places within a body to stiffen it. By placing three crease lines parallel to each other with equal spacing and folding them in an inside-outside-inside fashion, one can create vertical beams between two horizontal planes. Such T-fold are implemented to strengthen the box that forms the robot body. To save time and effort in the assembly process, inner T-folds are locked with the aid of tab-and-slot fasteners in each of the T-folds. These tab-and-slot fasteners stiffen the body structure and prevent the T-folds from buckling. Moreover, the T-folds are extended to lock into the side walls of the body as well, which play a key role in order to prevent the body from distorting under transverse forces and torsion. Increased rigidity and robustness of the main frame of the robot enables C-Quad to carry up to $220 \mathrm{gr}$ in addition to its weight, which gives the robot a payload capacity of more than $450 \%$ of its total weight.

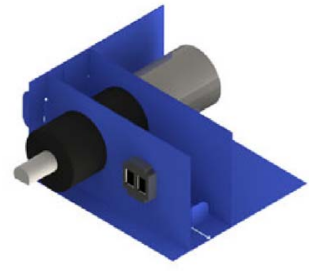

(a)

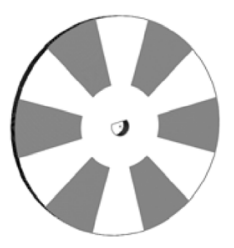

(b)
Fig. 2. (a) T-folds that contain DC motor and IR sensor housings. (b) Cam shaft encoder.

As an additional advantage of the T-folds, motor housings are built in as holes to the T-folds, which hold the motors tightly in-place within the body frame of the robot without

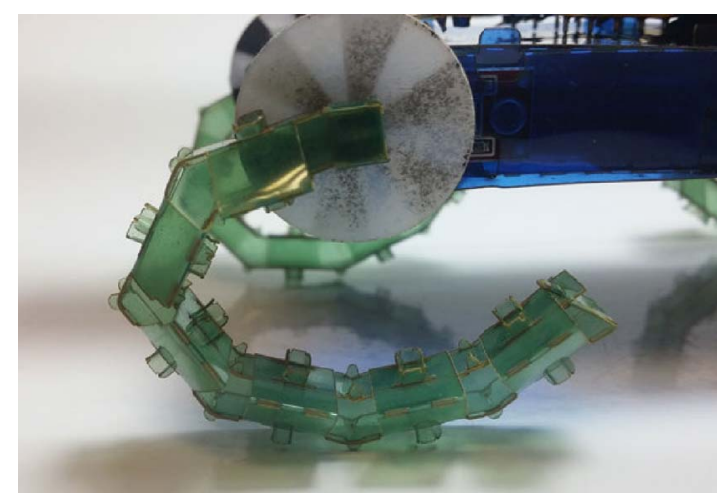

Fig. 3. The foldable C-shaped leg design.

requiring any supplementary housing. A pair of parallel Tfold structures are used to hold the DC motors and the IR sensors, which are located next to the DC motor as shown in Fig. 2(a). By placing DC motors and IR sensors within these structure, a separate encoder disk that will be used for the speed control of the motor is designed. The encoder disk rotates $360^{\circ}$ with the legs (Fig. 2(b)).

\section{B. C-Shaped Leg Design}

In order to obtain fast and durable locomotion, a variety of leg structures are studied. All four legs are designed as compliant C-shaped structures instead of wheels, which can be machined onto and folded from one complete sheet in order to meet the idea of ease of manufacturing and assembly. The fact that the $\mathrm{C}$-shaped legs are being produced by folding from a single thin sheet material makes them very light weight compared to regular wheels. By definition, leg structure are required to have robustness in order to be able to provide the desired locomotion and carry the weight of the robot complete with the battery and electronic boards. Moreover, C-shaped leg designs prevent stall cases during locomotion over obstacles, a problem that can be experienced in a small-wheeled robot [20]. In the beginning of the design process, a regular C-shaped leg (similar to those in [5] and [20]) is tried. The standard version of the C-shaped leg led to extremely imbalanced body during the locomotion. The main reason for this imbalance is the fact that the shape does not stay at a constant height with respect to the body while the leg is in contact with the surface.

Consequently, a modified C-shaped leg is designed such that the leg surface that contacts the ground would trace a circle whose center is located at the motor hub (Fig. 3). Placing the center of the C-shaped leg to the motor hub and sweeping the leg surface to the constant radii increases the contact time of the leg (Fig. 4). By increasing the total contact time between the leg and the ground and avoiding changing height of the body during contact, the undesirable motion of the body structure is prevented. These new design of the $\mathrm{C}$-shaped legs offers a fairly fast and robust locomotion for the C-Quad.

\section{Actuation, Electronics and Power}

In order to mimic a walking-like motion, instead of increasing the number of joints, manufacturing and assembly 


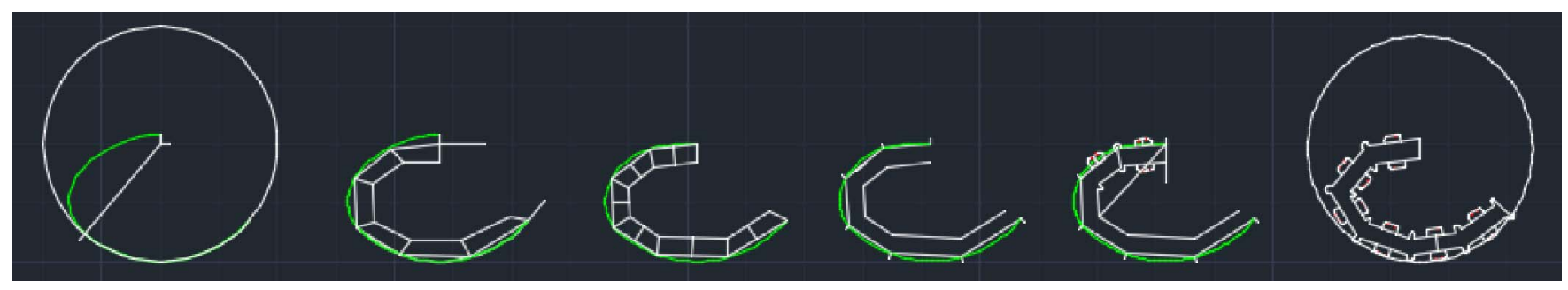

Fig. 4. Design iterations of C-shaped legs.

times; C-Quad exploits a set of four C-shaped compliant legs where each leg has 1 DOF, driven by one motor. To actuate the C-Quad's legs, four DC motors (Pololu sub-micro plastic planetary gearmotor with 136:1 gear ratio) are used. These motors are very small and lightweight, however they do not have encoders. The motors also do not run at the same speed in an open-loop operation with same input voltages. In order to overcome this issue, infrared reflectance sensors (Pololu, QTR-1A) coupled with custom rotational encoder disks are utilized. In order to drive the motors a circuit is designed with a voltage regulator, motor drivers and passive components required to get the regulator and drivers function properly. The sensor inputs are read into an Arduino Pro Micro and control loop is formed. Arduino then sends PWM signals to the designed motor driver circuit (that also powers the sensors), which sends the appropriate voltages to the DC motors. In order to control the robot remotely, an HC06 bluetooth serial module for wireless communication is attached to the Arduino controller. The complete electronics and actuators are powered by two $3.7 \mathrm{~V} 150 \mathrm{mAh} \mathrm{LiPo}$ batteries.

\section{MANUfACtURING AND ASSEMBLy}

Throughout the manufacturing process of C-Quad, thick polymer or polyimide films and PET sheets are considered as the base material in order to achieve a foldable robot which is easy to build. Although the thickness of the material directly increases the rigidity, it decreases the durability while folding because the creases are exposed to greater plastic deformation in thicker sheets. Besides, thinner films lead to relatively easier cutting, folding and assembly. Thus, 100-micrometer-thick PET sheets are used to manufacture C-Quad in order to exploit these advantages.

A total of two boards are designed for the C-Quad, one is the power board that has the voltage regulator on and connects to the battery and the sensors and the second board that has the motor drivers and the Arduino connector on. These boards are fabricated from flexible copper-clad polyimide films (Dupont, Pyralux) using simple PCB fabrication methods. The flexibility of the boards enable us to bend them at connection locations and make electrical connections to sensors and between the boards easier. In addition, the flexible PCB material is very light, and combined with the surface mount components selected, the custom boards only weigh a few grams.

The manufacturing process is initialized by cutting the 2D AutoCAD drawings of the body (Fig. 5(a)) and the leg designs (Fig. 5(b)) from 100-micrometer-thick PET sheets using a Universal Laser Systems VLS 6.60 laser engraver. In order to prevent the unfolded structures from the dislocating during cutting and getting damaged, it is not fully separated from the sheet material.

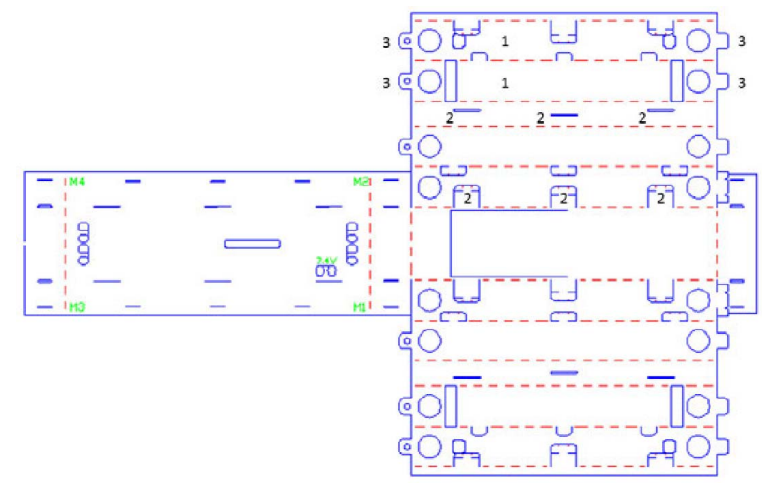

(a)

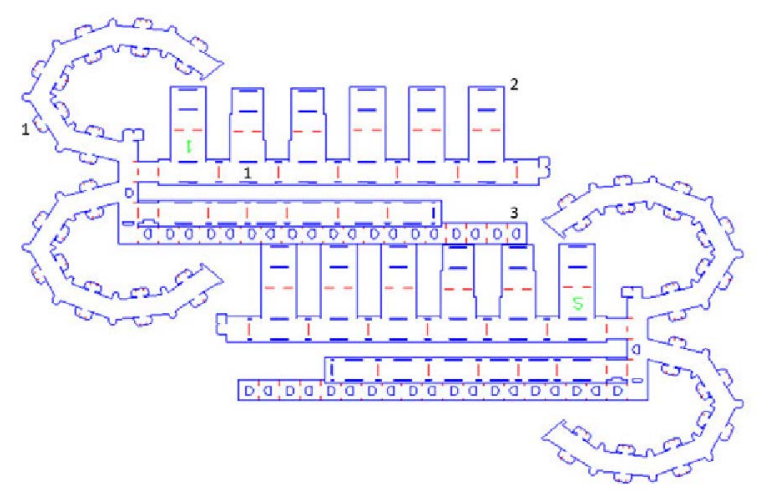

(b)

Fig. 5. (a) 2D AutoCAD drawings of the body where blue lines represent the full cuts and red lines represent the folding lines. (b) 2D AutoCAD drawings of the C-shaped leg design.

At the beginning of the assembly process, T-folds that are shown as Fig. 5(a) - 1 are folded by placing the IR sensors and DC motors in-place and locked with the double sided tab-and-slot fasteners, which can also be seen as Fig. 5(a) 2. In order to assemble the robot in a fast and easy fashion, the extended tabs are locked to the front and back of the body structure as highlighted as Fig. 5(a) - 3. At this point, small pins are inserted to the holes in the extended tabs to hold the entire body together during the assembly process. After placing the motors to their housings and electronic board with sensor connections inside the body frame of the C-Quad, the upper part of the body is fastened with the aid of extended tabs and U shaped locks.

After the body structure is assembled, each one of the leg design are locked with the aid of tabs (Fig. 5(b) - 1) and slots (Fig. 5(b) - 2). Leg structures are connected to the 
motor shaft along with the cam encoder disks with the aid of the holder folds (Fig. 5(b) - 3) designed to have a tight fit with the motor shaft. Lastly, the batteries are placed inside the body through the hatch located beneath the body, and the electronics are placed on top of the body. Without any components, the frame of the C-Quad weights 4.1 grams. After the final assembly, the robot weighs 38 grams and has a width of $70 \mathrm{~mm}$, height of $39 \mathrm{~mm}$ and a length of 103 $\mathrm{mm}$.

\section{Control Strategy}

\section{A. Control Problem}

Conventionally miniature legged robots try to utilize as few actuators as possible that will make the robot walk straight and steer in order to avoid synchronization issues of the legs and decrease the total weight and size of the robot. On the other hand, robots with C-shaped legs utilize one actuator per leg, which rotates the leg from the tip of the ' $\mathrm{C}$ ' shape. Among these robots, RHex uses motors with encoders whereas the actuation method for the Maryland robot is magnetics, hence the synchronization is guaranteed by using an external magnetic field.

Since C-Quad is a miniature quadruped with C-shaped legs, both approaches were discussed during the development phase. Even though coupling two legs and driving the whole robot using two actuators is possible using gearboxes and drive shafts, this approach would have mechanically lock the gait in place. However, RHex demonstrated that by individually controlling the C-shaped legs, the robot can perform interesting maneuvers and can even jump. Because of this reason, we selected to use one actuator per leg, however this choice is mostly motivated by our future plans of performing gait studies and interesting maneuvers with the C-Quad.

Due to the partially-circular shape of the legs, the four individually-actuated legs of the C-Quad make the robot move in a manner between a car and a four-legged animal. If the legs run at the same phase, the motion resembles a bouncy car, whereas if there are phase differences between the legs, the gait can change between a variety of horse gaits such as walk, trot, gallop, or canter. The current gait of the robot is not controlled precisely, we only implemented a speed controller. Due to small errors in the controlled speeds, the robot changes its gait during operation because the legs go in and out of phase. The individual speed control of the legs are achieved via custom made encoders and IR reflectance sensors. Our experiments at different leg speeds showed that C-Quad can gain its maximum speed when the motors are run at $30 \%$ duty cycle; which is $27.7 \mathrm{~cm} / \mathrm{sec}$ that corresponds to 2.7 Bodylengths/sec. This is under optimum traction conditions where there is only very little slippage between the legs and the ground.

\section{B. Custom Encoder Design and Control Input Signal}

Considering the control problem, an encoder was necessary to obtain the speed data of the legs. To keep the weight and size of the robot at minimum, one of the smallest DC motors in the market was selected. These small DC motors do not have a back shaft or an encoder. Therefore, we needed to design our own encoders and mount them on the same shafts with the legs. To be able to mount both the encoders and the legs on a single miniature motor shaft, the thickness of the encoder had to be limited. This constraint eliminated the use of a $3 \mathrm{D}$ encoder which could provide more data points to the controller. More data points could be used to control not only the speed of the motors but also the positions as well. However, to simply make the robot walk or turn, the experiments showed that we do not need sensitive position control of the legs. Cam shaft encoders and speed control of the motors are sufficient for an acceptable locomotion performance.

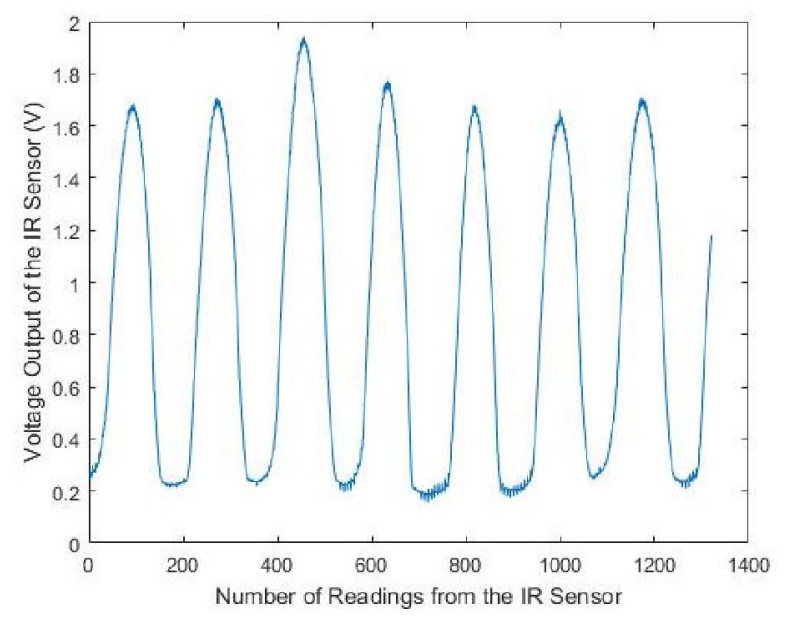

Fig. 6. Sample Analog Output from the IR sensors.

There are six black and six white bands on the customdesigned encoder disk. The infrared reflectance sensors were used to differentiate between the white and black bands on the encoder. The IR sensors were integrated to the body of the robot at an optimum distance to the encoders since the analog data from the sensors vary considerably with the distance. The analog output signal of the IR sensors had a peak every time a black band passed by the sensor. The peak and the valley points of the data are processed with a digital filter. The filter was necessary because as seen in Fig. 6, the data can be quite noisy especially in the valleys. The digital filter eliminates the possibility of detection of more than one data point on a single peak or a valley. The filter implemented is a simple low-pass filter with the addition that if the time between the data points are smaller than a determined value which depends on the assigned speed of the motors, then the data point is considered as faulty and eliminated. After the data is cleared from noise, the rate of the peaks was used to determine the speed of the motors. Fig. 6 represents a sample analog output data from the IR sensor.

\section{PD Speed Controller}

A PD controller was used to control the speed of each individual motor. The speed of the motors is proportional to the supplied voltage. To be able to drive four motors in both forward and backward directions, two L293d motor 


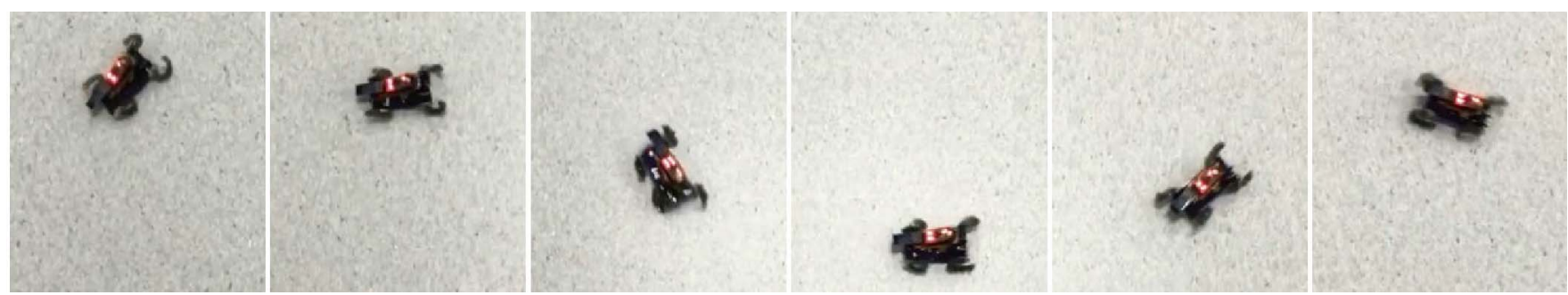

Fig. 7. Controlled steering of the C-Quad.

controller ICs were used. By creating PWM signals on Arduinos available digital pins, a duty cycle for each motor can be created. Since all the motors have different responses, the duty cycles need to be adjusted and may be different for each motor. Initially a duty cycle is given to run the motors and by using the feedback from the infrared sensors, the duty cycle of each motor is updated continuously to achieve a certain and constant speed. The Arduino collects the top and bottom data points of the analog signal from the IR sensors and measures the time between two consecutive data points to determine the frequency. The algorithm feeds this data to the PD controller to maintain a desired speed. For straight walking, each of the motors are adjusted to same speed and for turning operations their speeds are varied accordingly. Experiments showed that there were no steady state errors observed with the PD controller and this makes an integral term of a PID controller unnecessary, hence it was not used.

\section{Wireless Control}

In order to demonstrate the capabilities of the robot with ease, wireless remote control is needed. In order to achieve this, an HC-06 Bluetooth module was used to give the robot directions wirelessly. This module can work in two channels, it receives commands from the user for locomotion and steering and it also sends the user necessary experimental data about robot's control signals. This makes it easier to analyze the data and debug the code when the robot is operating on the ground but it is especially important when the external sensors that can be added to C-Quad in the future is considered. The wireless connection makes it possible for C-Quad to send back crucial data of its surroundings such as temperature, humidity, pressure, etc. in the future.

\section{LOCOMOTION RESULTS}

\section{A. Speed and Power Performance}

C-Quad has an ability to reach a speed of 2.7 Bodylengths/sec at its maximum speed with duty cycle of $30 \%$. Due to the newly proposed design of the C-Shaped legs, locomotion of the robot becomes smoother compared to the classic ' $C$ '-shaped legs. With the aid of the new design, total contact time of the legs with the ground or obstacle is increased. Besides, the vertical movement of the center of gravity of the C-Quad's body frame is reduced by the new C-Shaped legs. A demonstration of the straight walk of the robot is included in the video accompanying the manuscript.

The robot can operate for approximately 30 minutes on 2 fully charged $3.7 \mathrm{~V} 150 \mathrm{mAh}$ batteries when walking around on flat surface continuously.

\section{B. Steering}

The C-Quad, as a miniature robot, is expected to work inside confined spaces where there are usually limited space for steering operations. By considering this, the C-Quad is equipped with four actuators that allow it to perform tight turns. By rotating the motors on different sides of the robot in opposing directions, C-Quad can perform a zero-radius-turn. By switching the directions of the rotation on both sides, the robot can be tightly turned right or left. Additionally, C-Quad can turn at different turning radii by adjusting the relative speeds of left and right motors. Videos about steering and zero-radius-turns are included in the video accompanying the manuscript. A series of screen shots demonstrating the robot's steering capability is shown in Fig. 7.

\section{Locomotion Over Obstacles}

C-Quad should be able to operate on rough terrain where it is likely to encounter small obstacles. So far, other robots with C-shaped legs proved that this leg shape is very beneficial for rough terrain locomotion. We have conducted experiments to see how high of an obstacle can C-Quad scale. Our tests showed that the maximum height that the C-Quad can climb is $25 \mathrm{~mm}$, which is more than $64 \%$ of its height. The speed controller also helps the locomotion over obstacles. Once the motors are faced with resistance, they slow down and in order to maintain the required speed, the power going to the motor is increased. This increases power arriving the leg, which grips to the surface. This process works independently on all 4 legs and thus it provides the maximum traction to the surface. C-Quad's performance on locomotion over obstacles are demonstrated in the accompanying video and a series of screen shots showing C-Quad climbing to a 2.5-cm-obstacle is shown in Fig. 8.

\section{CONCLUSion And Future Work}

In this work, we introduced a foldable and miniature fourlegged robot, the C-Quad, whose body and legs are made out of PET sheets. To reduce the assembly time and also improve the stiffness of the robot, the practical T-shaped folds are utilized. The legs of the robot are individually actuated which makes it possible for the robot to complete difficult locomotion tasks such as zero-radius-turns. The custom-made encoders are used in speed control of the legs. The robot weighs about 38 grams including batteries and it can work non-stop nearly half an hour with a full charge. C-Quad has the ability to reach a maximum speed of 2.7 Bodylengths/sec when the motors work around 30 percent duty cycle and without gait control. The remaining power of 


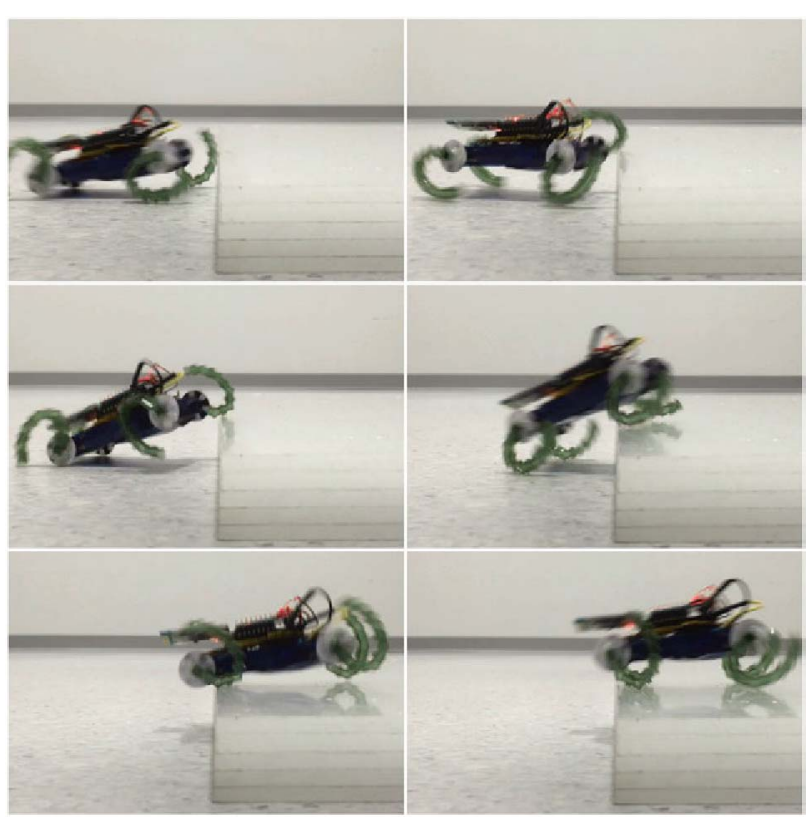

Fig. 8. C-Quad locomotion over obstacle of $2.5 \mathrm{~cm}$.

the robots are useful especially for the climbing operations and/or on rough terrain where algorithm can optimize the duty cycle of the motors to deliver more power when needed. One of the special qualities of the C-Quad is its foldable legs. The C-shaped legs can be cut from a PET-Sheet and can be folded into a fully functioning leg. C-Quad is also equipped with a Bluetooth transceiver which can receive control signals and send data back.

Even though the actuation and the design of the robot is sufficient to perform the mentioned tasks in the paper, leg slip issues remain. To reduce the problems caused by slipping, the robot could be equipped with an inertial measurement unit(IMU) in the future. The IMU can be used to have a more robust controller which can correct the direction of the robot if slip occurs. The IMU can also be used to improve the leg synchronization and gait locomotion so that the robot has more stable movement which would reduce the momentary stresses in the body and the legs of the robot, increasing the lifetime of the robot. As another future work, C-Quad will be utilized to perform gait studies on different terrain types. In order to perform this study, the encoders will be improved to also provide position control.

\section{ACKNOWLEDGMENTS}

The authors would like to thank every group member of Bilkent Microrobotics Laboratory for their assistance throughout this project. This work is funded by the Scientific and Technological Research Council of Turkey (TÜBİTAK) grant no. $115 \mathrm{C} 107$.

\section{REFERENCES}

[1] O. Ozcan, A. T. Baisch, D. Ithier, and R. J. Wood, "Powertrain selection for a biologically-inspired miniature quadruped robot," in 2014 IEEE International Conference on Robotics and Automation (ICRA). IEEE, 2014, pp. 2398-2405.

[2] R. Wood, S. Avadhanula, R. Sahai, E. Steltz, and R. Fearing, "Microrobot design using fiber reinforced composites," Journal of Mechanical Design, vol. 130, no. 5, p. 052304, 2008.
[3] J. Whitney, P. Sreetharan, K. Ma, and R. Wood, "Pop-up book mems," Journal of Micromechanics and Microengineering, vol. 21, no. 11, p. $115021,2011$.

[4] J. M. Bustillo, R. T. Howe, and R. S. Muller, "Surface micromachining for microelectromechanical systems," Proceedings of the IEEE, vol. 86, no. 8, pp. 1552-1574, 1998.

[5] R. S. Pierre and S. Bergbreiter, "Gait exploration of sub-2 g robots using magnetic actuation," IEEE Robotics and Automation Letters, vol. 2, no. 1, pp. 34-40, Jan 2017.

[6] A. M. Hoover, E. Steltz, and R. S. Fearing, "Roach: An autonomous 2.4 g crawling hexapod robot," in Intelligent Robots and Systems, 2008. IROS 2008. IEEE/RSJ International Conference on. IEEE, 2008, pp. 26-33.

[7] D. W. Haldane, K. C. Peterson, F. L. G. Bermudez, and R. S. Fearing, "Animal-inspired design and aerodynamic stabilization of a hexapedal millirobot," in Robotics and Automation (ICRA), 2013 IEEE International Conference on. IEEE, 2013, pp. 3279-3286.

[8] P. Birkmeyer, K. Peterson, and R. S. Fearing, "Dash: A dynamic $16 \mathrm{~g}$ hexapedal robot," 2009 IEEE/RSJ International Conference on Intelligent Robots and Systems, pp. 2683-2689, Oct 2009.

[9] A. T. Baisch, O. Ozcan, B. Goldberg, D. Ithier, and R. J. Wood, "High speed locomotion for a quadrupedal microrobot," The International Journal of Robotics Research, vol. 33, no. 8, pp. 1063-1082, 2014.

[10] C. D. Onal, R. J. Wood, and D. Rus, "Towards printable robotics: Origami-inspired planar fabrication of three-dimensional mechanisms," in Robotics and Automation (ICRA), 2011 IEEE International Conference on. IEEE, 2011, pp. 4608-4613.

[11] M. Salerno, K. Zhang, A. Menciassi, and J. S. Dai, "A novel 4-dof origami grasper with an sma-actuation system for minimally invasive surgery," IEEE Transactions on Robotics, 2016.

[12] E. Vander Hoff, D. Jeong, and K. Lee, "Origamibot-i: A threadactuated origami robot for manipulation and locomotion," in 2014 IEEE/RSJ International Conference on Intelligent Robots and Systems. IEEE, 2014, pp. 1421-1426.

[13] Y. Mulgaonkar, B. Araki, J.-s. Koh, L. Guerrero-Bonilla, D. M. Aukes, A. Makineni, M. T. Tolley, D. Rus, R. J. Wood, and V. Kumar, "The flying monkey: A mesoscale robot that can run, fly, and grasp," in 2016 IEEE International Conference on Robotics and Automation (ICRA). IEEE, 2016, pp. 4672-4679.

[14] C. D. Onal, R. J. Wood, and D. Rus, "An origami-inspired approach to worm robots," IEEE/ASME Transactions on Mechatronics, vol. 18, no. 2, pp. 430-438, 2013.

[15] K. Zhang, C. Qiu, and J. S. Dai, "Helical kirigami-enabled centimeterscale worm robot with shape-memory-alloy linear actuators," Journal of Mechanisms and Robotics, vol. 7, no. 2, p. 021014, 2015.

[16] M. Agheli, S. G. Faal, F. Chen, H. Gong, and C. D. Onal, "Design and fabrication of a foldable hexapod robot towards experimental swarm applications," in 2014 IEEE International Conference on Robotics and Automation (ICRA). IEEE, 2014, pp. 2971-2976.

[17] C. D. Onal, M. T. Tolley, R. J. Wood, and D. Rus, "Origami-inspired printed robots," IEEE/ASME Transactions on Mechatronics, vol. 20, no. 5, pp. 2214-2221, 2015.

[18] S. T. Kalat, S. G. Faal, U. Celik, and C. D. Onal, "Tribot: A minimallyactuated accessible holonomic hexapedal locomotion platform," in Intelligent Robots and Systems (IROS), 2015 IEEE/RSJ International Conference on. IEEE, 2015, pp. 6292-6297.

[19] S. Felton, M. Tolley, E. Demaine, D. Rus, and R. Wood, "A method for building self-folding machines," Science, vol. 345, no. 6197, pp. 644-646, 2014

[20] U. Saranli, M. Buehler, and D. E. Koditschek, "Rhex: A simple and highly mobile hexapod robot," The International Journal of Robotics Research, vol. 20, no. 7, pp. 616-631, 2001.

[21] C. Karakadioglu, M. Askari, and O. Ozcan, "Design and operation of miniaq: An untethered foldable miniature quadruped with individually actuated legs," in 2017 IEEE International Conference on Advanced Intelligent Mechatronics (AIM), July 2017, pp. 247-252. 